\title{
Allein gelassen
}

\author{
Mirjam Tanner, Franco Renato Gusberti
}

Dres. med., Mitglieder des Leitungsausschusses von ReMed

Kein ärztliches Team, mangelnde Unterstützung durch die Vorgesetzten, endlose Überstunden - schlechte Voraussetzungen, um seelisch gesund zu bleiben. Geschweige denn, um die Leidenschaft für die Medizin zu behalten. Der folgende Bericht zeigt, wie die ärztlichen Beratenden von ReMed, dem Unterstützungsnetzwerk für Ärztinnen und Ärzte, eine Assistenzärztin auf dem Weg aus ihrer Krise begleiten konnten.

Die junge Kollegin meldet sich per E-Mail via ReMed bei mir. Auf Wunsch und da geographisch machbar, vereinbaren wir einen Termin in meiner Praxis. Offen und erleichtert, Gehör zu finden, erklärt sie mir, warum sie Hilfe suche. «Ich bin Assistenzärztin, jetzt im vierten Jahr in meiner Weiterbildung zur Internistin. Schon das letzte Jahr war schwierig, weil ich mich von meinen Vorgesetzten und der Klinik nicht unterstützt gefühlt habe. Anstatt Hilfe zu erhalten, hatte ich immer mehr das Gefühl, im Stich gelassen zu werden und für die Konflikte unter den verschiedenen Spezialisten herhalten zu müssen. So habe ich meine Motivation zunehmend verloren, die Medizin gefällt mir immer weniger, und inzwischen bin ich richtig depressiv. So kann es nicht mehr weitergehen. Zum Glück hat mich die Personalabteilung auf ReMed aufmerksam gemacht.»

\section{Vom Selbstzweifel zur Depression}

Das letzte Assistenzjahr hatte für die Kollegin mit einer bösen Überraschung begonnen: Sie hatte erwartet, vom Weiterbildungsangebot des Universitätsspitals profitieren zu können, und war sehr enttäuscht über ihre Zuteilung in eine dem grossen Zentrumsspital angeschlossene Privatklinik. Sie wagte nicht, sich dagegen zu wehren - da sie sich unsicher fühlte und wie alle

\section{ReMed ist für Sie da}

Brauchen Sie oder jemand aus Ihrem Umfeld professionelle Hilfe? Wenden Sie sich an ReMed: Das Unterstützungsnetzwerk für Ärztinnen und Ärzte respektiert das Arztgeheimnis und berät Sie kompetent. Auch bei anderen beruflichen und persönlichen Krisen kann ReMed Ihnen Lösungswege aufzeigen. Dieses Angebot gilt auch für Personen aus dem Umfeld von Ärztinnen und Ärzten. 24 Stunden amTag. Die ärztlichen Beratenden melden sich innerhalb von 72 Stunden: www.swiss-remed.ch, help[at]swissremed.ch, Tel. 0800073633. ihre Kollegen fürchtete, dass eine Beschwerde in der Gesamtbeurteilung auf sie zurückfallen würde. Dies, obwohl ihre Feedbacks und Qualifikationsgespräche bisher stets positiv ausgefallen waren.

Im neuen Umfeld haben die Zweifel an ihren eigenen Fähigkeiten zugenommen. Sie berichtet, dass sie während des Studiums nach einer misslungenen Prüfung

"Ich hatte das Gefühl, im Stich gelassen zu werden und für die Konflikte unter den verschiedenen Spezialisten herhalten zu müssen.»

schon einmal an einer depressiven Phase gelitten habe. Professionelle Hilfe habe sie bisher nie beansprucht - aus Scham. Erst seit Kurzem nehme sie erstmals psychotherapeutische Hilfe in Anspruch. Ihren Psychiater sehe sie nur einmal im Monat, und er kümmere sich vor allem um den Medikamentenblutspiegel eines Antidepressivums, das ihr nicht helfe.

\section{Ausgelaugt und ungehört}

Der auferlegte Wechsel an die neue Rotationsstelle habe ihr den Rest gegeben. Das Zentrumsspital stellt der betreffenden Privatklinik halbjährlich einen Assistenzarzt zur Verfügung. "Dort arbeitet man sehr alleine. Ausser dem Chef gibt es keine Ansprechperson für medizinische Fragen.» Anstatt sich im überschaubareren Milieu sicherer zu fühlen, kämpft die Kollegin nun gegen blockierende Ängste und Gefühle der Überforderung. «Ich fürchte immer stärker, schwerwiegende medizinische Fehler zu machen, und kontrolliere alle meine Verordnungen noch und noch. So bin ich in einen Teufelskreis aus Überstunden geraten.» Die stets wachsenden administrativen Arbeiten verschlimmern die zeitliche Belastung zusätzlich.

Aus organisatorischen Gründen sei es ihr nicht mehr möglich gewesen, an den Weiterbildungen teilzuneh- 


\section{Coaching-Gruppen}

Die Coaching-Gruppen bieten den idealen Rahmen für einen vertrauensvollen Austausch über sämtliche schwierigen beruflichen Themen, z.B. den Umgang mit Kolleginnen und Kollegen sowie Vorgesetzten, Ängsten oder Belastungsgrenzen. Das Ziel der Coaching-Gruppen ist es, unter fachlich kompetenter Anleitung in ein kollegiales Gespräch zu kommen und sich gegenseitig zu bestärken. Das Angebot richtet sich an Assistenz- und Oberärztinnen und -ärzte am Spital und in Praktika sowie an niedergelassene Medizinerinnen und Mediziner. Es schliesst Teilnehmende sämtlicher Fachrichtungen ein. Zurzeit werden Coaching-Gruppen in Bern, Zürich und Chur durchgeführt. Genauere Informationen finden Sie unter www.swiss-remed.ch $\rightarrow$ Weitere Angebote $\rightarrow$ Coaching-Gruppen.

men. Jetzt befürchte sie, dass ihr dieses halbe Jahr nicht einmal für ihre Weiterbildung angerechnet werde. «Ich habe versucht, meinen Chef darauf anzusprechen, ihm gesagt, dass es mir nicht gut geht ... Aber die Nachricht ist nicht angekommen.» Sie schlafe schlecht, habe Mühe, sich zu konzentrieren, sei immer nervöser. Kurz, sie fühle sich nicht mehr in der Lage weiterzuarbeiten.

Korrespondenz:

Mirjam Tanner

mirjam.tanner[at]hin.ch

Franco Renato Gusberti

frgusberti[at]hin.ch

\section{Atempause und Gespräche}

Um diese Situation zu klären, gilt es vorerst, das Verhältnis zum bisherigen Psychiater zu regeln. Anschliessend bespreche ich mit der Kollegin das weitere Vorgehen,

d.h., wie wir zusammen eine psychiatrischpsychotherapeutische Behandlung unter Einbezug eines anderen Antidepressivums und einer kurzfristigen Krankschreibung gestalten können. Ich betone für die Phase der Arbeitspause, wie wichtig ein oder sogar zwei wöchentliche Gespräche sind, um ihre aktuelle tägliche Situation intensiv zu analysieren und zu besprechen. Dieser Austausch kann entscheidend dazu beitragen, dass sie diese schwierige Zeit durchsteht und kon-

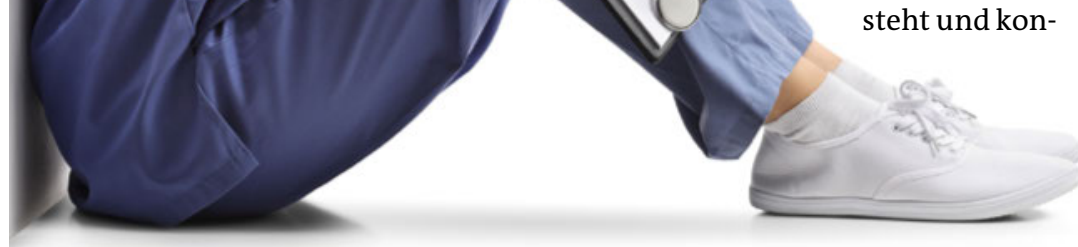

Wenn die Weiterbildung zu viel Kraft raubt, statt die Freude am Arztberuf zu stärken: Das erfahrene ärztliche Beratungsteam von ReMed, dem Unterstützungsnetzwerk für Ärztinnen und Ärzte, hört zu und hilft weiter. krete Lösungen ihrer Probleme erarbeiten kann. Die Kollegin kann mich im Notfall auch telefonisch erreichen. Weiter verweise ich sie auf den Rechtsberater ihres Kantons vom Verband Schweizerischer Assistenzärtzinnen und -ärzte VSAO, der sich in Sachen Anerkennung der Weiterbildung für sie einsetzen und ihre Rechte als Assistenzärztin verteidigen kann.

Doch nun hat die junge Kollegin Angst, ein solches Vorgehen schade ihrem Ruf bei den Vorgesetzten, beim Spital und bei späteren Anstellungen. Es ist nicht leicht, mit ihr einen gangbaren Weg zu finden, der ihr schliesslich nach einigen Wochen hilft, aus der vertrackten Situation herauszukommen. Sie hat seither die Arbeit wieder aufgenommen, lernt nach und nach, sich besser zu wehren, und entwickelt wieder mehr Freude an ihrem Beruf.

\section{ReMed-Intervisionen für Erstberatende und Netzwerkmitglieder}

Neben den Unterstützungsangeboten für ratsuchende Ärztinnen und Ärzte führt ReMed seit 2009 auch regionale Intervisionen zum Erfahrungsaustausch für Kolleginnen und Kollegen durch, die Ärztinnen und Ärzte als Patienten betreuen. Diese ermöglichen Vernetzung und Bildung von Peer-Groups (jeweils 6-8 Teilnehmer, 2-3 Treffen pro Jahr), welche gemeinsam Fallfragen zu Mentoring, Coaching, Beratung, Therapie oder anderen Aspekten (juristisch, versicherungsrechtlich etc.) erarbeiten. Setzen Sie sich mit uns in Verbindung, nehmen Sie an einer Sitzung teil und lernen Sie unsere Arbeit kennen. Kontakt und Anmeldung Dr. med. Sabine Werner, Mitglied Leitungsausschuss ReMed, dr.s.werner[at]hin.ch.

Mögliche nächste Daten: 7. Juni, 21. Juni, 20. September, 25. Oktober und 15. November 2018, jeweils 14-18 Uhr; in Zürich.

\section{Druck und Stigmatisierung kontern}

In ärztlichen Kreisen werden medizinisch indizierte Arbeitsunterbrüche oft schlecht akzeptiert, auch wenn sie längerfristig durchaus sinnvoll und auch für das Spital schliesslich vorteilhaft sind. Kurzfristiges Denken unter Stress beherrscht das Arbeitsklima, und es besteht offenbar manchmal auch eine Tendenz zur Stigmatisierung. Betroffene wie Vorgesetzte interpretieren eine depressive Phase fälschlicherweise häufig als Schwäche oder Unfähigkeit. Woher kommt das wohl? Was tun? Wir kommen nicht umhin festzustellen, dass heute das ganze System unter zu hohem Druck steht. In der Folge verlieren junge Ärztinnen und Ärzte zunehmend die Motivation für den Beruf und haben Angst, nicht mehr standhalten zu können. Wie viele junge Kolleginnen und Kollegen bleiben dann noch für die Zukunft in der Medizin? Gegenmassnahmen sind dringend geboten.

Bildnachweis

() Ljupco | Dreamstime.com 